\title{
Condutas para 0 trabalho de parto prematuro
}

\author{
Management of preterm labor
}

Roberto Eduardo Bittar ${ }^{1}$, Mário Henrique Burlacchini de Carvalho², Marcelo Zugaib

\section{RESUMO}

O parto prematuro é a principal causa de morbidade e mortalidade neonatal. Cerca de $75 \%$ dos nascimentos prematuros são decorrentes do trabalho de parto espontâneo. A patogenia do parto prematuro espontâneo é complexa e o sucesso do seu manejo depende da pesquisa cuidadosa dos riscos maternos e fetais para dar continuidade ou não à gestação. $\mathrm{O}$ objetivo deste artigo é fazer uma revisão do diagnóstico do trabalho de parto prematuro, tocólise, corticoterapia, tratamento antibiótico e assistência ao parto prematuro.

PALAVRAS-CHAVE: Trabalho de parto prematuro

\section{ABSTRACT}

Preterm delivery is the most common cause of neonatal morbidity and mortality. About $75 \%$ of preterm births follow preterm labor. The pathogenesis of spontaneous preterm birth is complex and its clinical management is based on a careful assessment of the risks for mother and infant and on continuing the pregnancy versus delivery. The goal of the present article is to review the diagnosis of preterm labor, the tocolytic treatment, glucocorticoid therapy, antimicrobial treatment, and management of progressive preterm labor.

KEYWORDS: Labor, premature

\section{Introdução}

O nascimento prematuro é um dos maiores problemas ainda não solucionados da Obstetrícia. Apesar de as estratégias de prevenção durante o pré-natal e da assistência neonatal terem evoluído muito nos últimos anos, a sua incidência, morbidade e mortalidade neonatal continuam elevadas. Além disso, suas complicações estão entre as principais causas de morte no primeiro ano de vida da criança ${ }^{1,2}$.

Além dos fatores de risco já sobejamente conhecidos e dificeis de serem controlados, tais como os de ordem socioeconômica, as infecções e as diversas complicações clínicas e obstétricas, nos últimos anos vem se somando a gestação gemelar, muitas vezes decorrente da gravidez em idade avançada e da maior oferta de técnicas de reprodução assistida. Além disso, o estresse emocional cotidiano da mulher moderna também vem contribuindo para o maior risco de parto prematuro.

Salienta-se que, em aproximadamente $75 \%$ dos casos, o parto prematuro é espontâneo, ou seja, a gestante inicia espontaneamente o trabalho de parto. Nos $25 \%$ restantes o parto prematuro é eletivo, isto é, a interrupção da gestação é realizada em decorrência de alguma complicação materna e/ou fetal ${ }^{3}$.

Vários são os aspectos polêmicos que envolvem a assistência ao trabalho de parto prematuro (TPP): o seu diagnóstico, as possíveis causas subjacentes, a decisão de se prolongar a gravidez por meio da tocólise, uso de corticosteróides, antibióticos e a assistência ao parto.

1 Professor Associado da Disciplina de Obstetrícia da Faculdade de Medicina, Universidade de São Paulo - USP - São Paulo (SP) - Brasil.

2 Assistente Doutor da Disciplina de Obstetrícia da Faculdade de Medicina, Universidade de São Paulo - USP - São Paulo (SP) - Brasil.

3 Professor Titular da Disciplina de Obstetrícia da Faculdade de Medicina, Universidade de São Paulo - USP - São Paulo (SP) - Brasil.

Clinica Obstétrica da Faculdade de Medicina, Universidade de São Paulo - USP - São Paulo (SP) - Brasil.

Correspondência: Roberto Eduardo Bittar

Av. Dr. Enéas de Carvalho Aguiar 255, 10 and. - 05403-000 - Cerqueira César-SP - Fax: (11) 4195-9190 - e-mail: roberto.bittar@hcnet.usp.br 


\section{Diagnóstico do trabalho de parto prematuro}

O diagnóstico correto do TPP nem sempre é fácil e classicamente baseia-se na presença de contrações uterinas regulares (pelo menos uma a cada 5 minutos) e persistentes, dilatação cervical igual ou superior a $1 \mathrm{~cm}$, esvaecimento cervical igual ou superior a $80 \%$ e progressão das alterações cervicais ${ }^{4}$. No falso trabalho de parto verifica-se apenas o aparecimento de contrações irregulares e sem coordenação, além da ausência de modificações importantes no colo uterino. Nos casos duvidosos é importante que a gestante permaneça em observação clínica por período mínimo de duas a três horas. Nestas situações também pode ser utilizado o teste da fibronectina fetal que, por apresentar valor preditivo negativo elevado (acima de 90\%), exclui os falsos trabalhos de parto, evitando as internações desnecessárias ${ }^{5,6}$.

A decisão de se prolongar a gestação requer a análise cuidadosa das condições materno-fetais. Diante de situações que tornem hostil o ambiente intra-uterino, ou que se agravem com a continuidade da gestação, não se deve inibir o trabalho de parto.

Caso se decida pela inibição das contrações uterinas, a gestante deve ser internada e mantida em repouso no leito. Constatada uma causa subjacente, esta deve ser tratada especificamente. Alguns exames complementares devem ser solicitados nesta ocasião: ultra-sonografia obstétrica - para os casos em que não há exame recente ou existam dúvidas em relação à idade gestacional e/ou crescimento fetal; cardiotocografia fetal desde que haja viabilidade fetal é imprescindivel a avaliação do bem-estar fetal; amniocentese para estudo do líquido amniótico - quando houver dúvida em relação à maturidade fetal ou à presença de corioamnionite; urina tipo I, cultura de urina, exame bacterioscópico e cultura do conteúdo vaginal - sempre devem ser solicitados em virtude da elevada incidência de infecções genitourinárias entre estes casos.

\section{Tocólise}

A inibição das contrações uterinas está indicada nas seguintes situações: período de latência do trabalho de parto, ou seja, dilatação cervical inferior a $3 \mathrm{~cm}$, e idade gestacional de 22 a 34 semanas, desde que o serviço possua bom nivel de atendimento neonatal. Nos locais onde os riscos para os recém-nascidos prematuros são maiores, a inibição deve ser estendida até 36 semanas. As contra-indicações para inibição das contrações são: morte fetal, sofrimento fetal, malformações fetais graves, restrição de crescimento, rotura prematura de membranas, corioamnionite, sindromes hemorrágicas, sindromes hipertensivas, diabete insulino-dependente ins- tável e outras doenças maternas em que há insuficiência placentária. É importante salientar que os riscos e beneficios devem ser avaliados cuidadosamente em cada caso. A tocólise mostra comprovado benefício clínico por curto período de tempo, ou seja, consegue adiar o parto, na maioria das vezes, por apenas 48-72 h, o que não deixa de ser importante para o uso do corticosteróide. Por outro lado, todos os uterolíticos, em maior ou menor grau, apresentam segurança discutivel em relação aos efeitos colaterais maternos e fetais.

\section{Hidratação parenteral}

Pode ser utilizado soro fisiológico e glicosado a 5\% IV, em partes iguais, totalizando $1.000 \mathrm{~mL}$, em uma hora. Embora tal esquema seja utilizado com freqüência, não há evidências de que seja útil em adiar o parto ${ }^{7}$. Por outro lado, a infusão de líquidos deve ser criteriosa por aumentar o risco de edema pulmonar com o uso posterior de uterolíticos, em especial com beta-agonistas.

\section{Inibição farmacológica}

\section{Beta-agonistas}

Entre as drogas beta-adrenérgicas destacamse: a terbutalina, o salbutamol, o fenoterol e a ritodrina, embora somente esta última tenha sido aprovada pela Food and Drug Administration dos EUA para inibir trabalho de parto. Estas drogas atuam em receptores beta-1 (coração e intestinos) e predominantemente beta-2 (miométrio, vasos sangüineos e bronquíolos) estimulando-os e determinando o relaxamento da fibra muscular uterina, por diminuição do cálcio livre no interior das células. O mecanismo envolvido neste efeito consiste na ativação da enzima adenilciclase, que catalisa a conversão do ATP em AMP-c. Este último, por sua vez, ativa a enzima proteínoquinase, responsável direta pela diminuição do cálcio intracelular. Portanto, estes fármacos agem em diversos órgãos, e no sistema cardiovascular são potencialmente perigosos. Além disso, atravessam a placenta, tendo sido descritos diversos efeitos colaterais no feto, entre os quais taquicardia, hiperinsulinismo, hipoglicemia, hipocalemia e hipotensão arterial.

O esquema terapêutico com a terbutalina é o seguinte: diluem-se 5 ampolas ( $1 \mathrm{amp}$. $=0,5 \mathrm{mg}$ ) em soro glicosado a $5 \%-500 \mathrm{~mL}$ e infunde-se por via intravenosa, iniciando com $2,5 \mu \mathrm{g} / \mathrm{min}$ (10 gts / $\mathrm{min})$. Aumentam-se $10 \mathrm{gts} / \mathrm{min}$ a cada $20 \mathrm{~min}$ até o máximo de $80 \mathrm{gts} / \mathrm{min}$. Obtida a dose minima capaz de cessarem as contrações, mantém-se o gotejamento por $24 \mathrm{~h}$. Caso as contrações não diminuam em 6 horas ou se a tocólise for necessária por mais de $24 \mathrm{~h}$, deve-se questionar a presença de corioamnionite ou insuficiência placentária. Após as 24 h de administração da dro- 
ga, diminuem-se 10 gts a cada 20 min até a suspensão total da mesma. Deve-se manter a paciente em repouso absoluto e sob vigilância por mais $24 \mathrm{~h}$ e, caso ocorra o retorno das contrações, utiliza-se novamente o esquema intravenoso.

Alguns cuidados devem ser tomados por ocasião do uso destas drogas: realizar eletrocardiograma materno prévio, controlar cuidadosamente o pulso e a pressão arterial, mantendo o pulso materno abaixo de 120 batimentos por minuto, auscultar periodicamente os pulmões e coração e monitorizar os batimentos cardíacos fetais. Devese salientar que os efeitos colaterais cardiovasculares, como o edema agudo de pulmões, são mais freqüentes em situações de hipervolemia materna, tais como no polidrâmnio, gestação gemelar e em pacientes submetidas à infusão de grande quantidade de líquidos. É importante destacar que diante da tocólise com beta-agonistas, a administração de liquidos não deve ultrapassar dois litros em $24 \mathrm{~h}$.

\section{Antagonista da ocitocina}

O antagonista da ocitocina (atosiban) é peptídeo sintético que age competindo com a ocitocina no seu receptor da célula miometrial e reduz os efeitos fisiológicos da ocitocina. Os estudos controlados e duplo-cegos de maiores casuísticas revelaram propriedades tocoliticas semelhantes aos beta-agonistas, porém com diminuição significante de efeitos colaterais maternos e perinatais ${ }^{8-10}$. Embora a nossa experiência atual ainda seja pequena, com apenas 23 casos estudados até o momento, os resultados confirmam os de estudos anteriores. Com o seu emprego conseguimos adiar o parto por duas semanas, em média, com efeitos colaterais maternos minimos e ausência de efeitos colaterais perinatais. $\mathrm{O}$ produto deve ser administrado em três etapas: inicialmente emprega-se uma ampola de 0,9 $\mathrm{mL}(7,5 \mathrm{mg} / \mathrm{mL})$ injetada diretamente na veia, durante um minuto; infunde-se uma solução IV contendo 2 ampolas de $5 \mathrm{~mL}$ em $90 \mathrm{~mL}$ de SG5\% com velocidade de $24 \mathrm{~mL} / \mathrm{h}(300 \mu \mathrm{g} / \mathrm{min})$ em $3 \mathrm{~h}$ e diminui-se o gotejamento da solução que sobrou da etapa anterior $(28 \mathrm{~mL})$ para $8 \mathrm{~mL} / \mathrm{h}(100 \mu \mathrm{g} / \mathrm{min}) \mathrm{du}-$ rante 3 h $30 \mathrm{~min}$. Com este esquema de 6 h $30 \mathrm{~min}$, utilizando-se uma ampola de 0,9 mL e duas ampolas de $5 \mathrm{~mL}$, geralmente consegue-se atingir a quiescência uterina. Entretanto, se após a utilização deste esquema ainda persistirem contrações uterinas compativeis com trabalho de parto, deverá ser mantida a mesma solução anterior na velocidade de $8 \mathrm{~mL} / \mathrm{h}(100 \mu \mathrm{g} / \mathrm{min})$ durante o período de 12 h $30 \mathrm{~min}$. Após o emprego da mesma as contrações devem ser reavaliadas para se saber se o esquema deverá ser repetido por mais 12 h 30 min. Deve ser salientado que a duração total do tratamento não deve exceder $48 \mathrm{~h}$.

\section{Sulfato de magnésio}

O sulfato de magnésio age como antagonista do cálcio na fibra muscular. Trata-se de uma alternativa para determinadas situações clínicas em que o beta-agonista não possa ser utilizado. Pode ser empregado na dose de $4 \mathrm{~g}$ diluído em soro glicosado a 10\%, infundido IV em $20 \mathrm{~min}$, como dose de ataque, seguido de 2 a 3 g/h até cessarem as contrações uterinas. A paciente deve ser cuidadosamente monitorizada em relação à diurese, freqüência respiratória e reflexos patelares. Além disso, deve-se avaliar a magnesemia materna a cada $6 \mathrm{~h}$. Apesar dos riscos potenciais, poucos efeitos colaterais maternos são observados quando a concentração sérica de magnésio é mantida em niveis terapêuticos (4 a $6 \mathrm{mEq} / \mathrm{L}$ - mg/dL). A hipermagnesemia fetal está relacionada à hiporreatividade e hipotonia.

\section{Inibidores de prostaglandinas}

Os inibidores de prostaglandinas atuam inibindo a enzima ciclooxigenase necessária à conversão de ácido araquidônico em prostaglandinas, e aparentemente são eficazes como uterolíticos, além de serem bem tolerados e de fácil administração. No entanto, são pouco utilizados pelos efeitos colaterais perinatais.

O esquema mais comumente empregado é o de uma dose inicial de $100 \mathrm{mg}$ por via retal, seguidos de $25 \mathrm{mg}$ por via oral a cada $6 \mathrm{~h}$, por um periodo de dois a três dias, para idades gestacionais inferiores a 34 semanas. Por atravessarem facilmente a placenta, inibem a sintese de prostaglandinas nos tecidos fetais. Como conseqüências podem ocorrer a enterocolite necrotizante, fechamento precoce do ducto arterioso, hipertensão pulmonar primária, oligoâmnio e hemorragia intracraniana. Em virtude destas intercorrências a ecocardiografia fetal e a ultra-sonografia devem ser realizadas com freqüência a fim de se detectarem precocemente sinais de constrição do ducto arterioso e oligoâmnio.

\section{Bloqueadores de canais de cálcio}

Inibem a entrada do cálcio extracelular através da membrana citoplasmática. As informações disponíveis ainda são escassas, mas alguns estudos relatam bons resultados na inibição do TPP ${ }^{11}$. Dos bloqueadores de canais de cálcio a nifedipina é a mais utilizada. É empregada na dose inicial de $30 \mathrm{mg}$ via oral, seguidos de $20 \mathrm{mg}$ via oral, a cada 8 horas. O efeito colateral materno mais comum é o enrubescimento facial, mas náuseas e cefaléia também ocorrem. Alguns estudos em animais sugerem que estas drogas reduzem o fluxo sangüíneo uterino e a oxigenação fetal. Tais efeitos colaterais fizeram com que os próprios fabri- 
cantes desencorajassem o seu uso para a tocólise. Portanto, ainda há necessidade de estudos clínicos controlados para determinar com maior precisão a aplicabilidade destas drogas no TPP.

\section{Manutenção pós-tocólise}

Não há evidências de que o emprego de betaagonistas por via oral na pós-tocólise impeçam a recorrência do TPP ou evitem o parto prematuro ${ }^{12}$. Baseando-se em estudos anteriores em que ficou demonstrado que a progesterona tem a função de manter a quiescência uterina, temos utilizado a progesterona natural micronizada na dose de 200 $\mathrm{mg} /$ dia, pela via vaginal. Entretanto, ainda não existem dados de literatura que confirmem os seus benefícios quando utilizada após a inibição do TPP ${ }^{13,14}$.

\section{Antibióticos}

O uso de antibióticos, na ausência de infecção, com o intuito de prolongar a gestação e evitar as complicações perinatais, carece de comprovação científica e, em alguns estudos, observou-se até aumento da morbidade perinatal ${ }^{15}$.

\section{Profilaxia da transmissão vertical do estreptococo do grupo $B$}

O estreptococo do grupo B (Streptococcus agalactiae) freqüentemente é encontrado na vagina e no reto das mulheres grávidas. Quando esta bactéria é transmitida da mãe para o feto, o que se dá na maioria das vezes durante o trabalho de parto e no parto, pode ocorrer septicemia neonatal, o que é mais comum e mais grave no prematuro. A profilaxia deve ser feita nos casos com cultura vaginal ou retal positiva ou desconhecida para o estreptococo. Igualmente, a presença do estreptococo na urina durante a gestação também indica a profilaxia. Dessa maneira, nos casos de TPP anteriores à $35^{\mathrm{a}}$ semana em que não se dispõe do resultado da cultura para estreptococo devese fazer a profilaxia, independente da escolha da via de parto ${ }^{16}$. Entretanto, havendo resultado de cultura negativa recente (menos de 5 semanas), a antibioticoterapia é desnecessária.

Para a profilaxia emprega-se a penicilina $G$ - 5 milhões UI - IV de ataque e 2,5 milhões UI - IV $4 / 4$ h até o nascimento, ou ampicilina - 2 g IV de ataque e $1 \mathrm{~g}$ IV $4 / 4 \mathrm{~h}$ até o nascimento. Diante de alergia à penicilina deve-se empregar a clindamicina $900 \mathrm{mg}$ IV $8 / 8 \mathrm{~h}$ até o nascimento. Em ambos os esquemas são necessárias pelo menos duas doses antes do nascimento.

\section{Corticoterapia}

A partir de 1972, quando Liggins e Howie empregaram a betametasona em mulheres grávidas e demonstraram a redução das complicações pulmonares em recém-nascidos prematuros, várias investigações têm demonstrado os beneficios da terapêutica antenatal com corticosteróides que, de maneira geral, incluem: redução de 40 a $60 \%$ de incidência de membrana hialina entre recémnascidos entre 28 a 34 semanas, menor gravidade da sindrome da angústia respiratória (SAR) quando presente, menor incidência de hemorragia intracraniana, maior sobrevida dos recémnascidos prematuros, com melhora na estabilidade circulatória e com necessidades reduzidas de oxigenação e de suporte ventilatório. Além disso, observam-se melhores respostas terapêuticas ao uso do surfactante neonatal quando a paciente faz uso do corticosteróide no período antenatal. Embora os corticosteróides antenatais não diminuam claramente a incidência de SAR em recémnascidos entre 24 e 28 semanas, parecem reduzir a gravidade do quadro e reduzir em mais de $50 \%$ o risco de hemorragias intraventriculares ${ }^{17,18}$.

Preconizamos o uso de corticosteróides entre e a $26^{\mathrm{a}}$ e $34^{\mathrm{a}}$ semana de gestação. Damos preferência à betametasona - $12 \mathrm{mg}$ IM ao dia com intervalo de $24 \mathrm{~h}$, num total de duas aplicações. Como segunda opção pode-se utilizar a dexametasona - $6 \mathrm{mg}$ IM de $12 \mathrm{em} 12 \mathrm{~h}$, num total de quatro aplicações. Em ambos os esquemas o efeito máximo se inicia em $24 \mathrm{~h}$. Geralmente optamos por um único ciclo de corticosteróide semanal, excepcionalmente dois, em virtude dos efeitos colaterais a longo prazo.

Apesar de, em 1995, o National Institute of Health (NIH) dos Estados Unidos da América ter chegado a um consenso favorável à administração de corticosteróides no período antenatal, dúvidas ainda persistem em relação aos efeitos adversos, tais como: maior incidência de infecções maternas e neonatais, supressão da adrenal fetal, alterações cardiotocográficas (diminuição da variabilidade dos batimentos cardiacos fetais), redução do perímetro cefálico e do peso ao nascer, má adaptação fetal à hipoxia e alterações de comportamento na infância. Assim, os efeitos dos corticosteróides não são específicos para o tecido pulmonar mas também têm influências sobre outros tecidos com divisão celular rápida, como demonstrado no tecido cerebral, intestinos, pâncreas e pele. Esta falta de especificidade é a base para o temor de prováveis efeitos deletérios para o concepto a curto ou a longo prazo. A partir de 1999 surgiram estudos experimentais em animais revelando deficiência de mielinização cerebral, alteração da função do eixo hipotálamo-hipofisário-adrenal e restrição de crescimento fetal quando foram utilizadas doses múltiplas de corticosteróide antenatal. Da mesma maneira, em humanos, embora as conclusões ainda sejam controversas, também sugerem supressão do eixo hipotálamo-hipofisário-adrenal, 
sepse neonatal, diminuição do crescimento somático e cerebral, retardo psicomotor e alterações do comportamento em crianças que na vida intra-uterina receberam acima de dois ciclos de corticóide. Tais efeitos foram mais graves quando se utilizou a dexametasona ${ }^{17-21}$.

\section{Assistência ao parto prematuro}

Trata-se de um dos temas mais relevantes e controversos da prematuridade. O momento do parto aumenta os riscos inerentes da prematuridade, tais como a maior suscetibilidade à acidose e ao trauma.

A boa assistência ao parto prematuro depende, antes de tudo, da experiência da equipe médica. Há necessidade, no acompanhamento do trabalho de parto, de pelo menos dois obstetras. Não se pode esquecer que em cerca de metade dos casos, o parto prematuro está associado a problemas clínicos e obstétricos que envolvem riscos, tais como a rotura prematura de membranas, gestação gemelar, placenta prévia, descolamento prematuro de placenta, hipertensão arterial grave, etc. Além disso, os procedimentos obstétricos no prematuro são mais dificeis de serem realizados. Os anestesistas devem possuir vivência com estes casos a fim de optarem pelo melhor tipo de anestesia. Assim, a integração da equipe constituída por obstetras, anestesistas, neonatologistas e enfermagem deve ser a melhor possivel. Um bom berçário e uma UTI neonatal adequada são fundamentais para que o trabalho de toda a equipe envolvida alcance o sucesso esperado.

Nos últimos anos observou-se aumento considerável da sobrevida dos prematuros. Sem dúvida, os cuidados intensivos neonatais colaboraram muito com isto, mas os obstetras também tiveram importante participação nestes resultados. O emprego mais freqüente do corticosteróide antenatal, os cuidados com a vitalidade fetal anteparto e intraparto e a escolha mais criteriosa da via de parto têm contribuído para a maior sobrevida dos prematuros.

\section{Escolha da via de parto}

Devem ser considerados os seguintes aspectos para se decidir sobre a melhor via de parto: idade gestacional (viabilidade), peso estimado do feto, apresentação fetal, condições do colo uterino, integridade das membranas amnióticas, possibilidade de monitorização fetal, experiência da equipe e condições do berçário.

O melhor tipo de parto no prematuro ainda é discutivel. Na literatura há carência de estudos prospectivos controlados, em virtude das inúmeras variáveis que fogem do controle. Não há dados convincentes que contra-indiquem o parto vaginal na apresentação cefálica fletida. Sendo assim, optamos pela via vaginal nas apresentações cefálicas fletidas, nas apresentações pélvicas com peso fetal estimado pela ultra-sonografia igual ou superior a $2.500 \mathrm{~g}$ e naqueles fetos considerados inviáveis. Ao se escolher a via vaginal para fetos viáveis é fundamental o controle da vitalidade com a cardiotocografia. Deve-se considerar que a interpretação da monitorização fetal é mais dificil no prematuro, principalmente se a gestante fez uso recente de uterolíticos que, sabidamente, influenciam nos batimentos cardiacos fetais.

Durante a assistência ao parto prematuro deve-se evitar o uso de analgésicos e tranqüilizantes ou sedativos, que deprimem a respiração. A analgesia do parto com anestesia combinada (raqui + peridural) deve ser instituída sempre que possivel para minimizar a reação de estresse em resposta à dor e à ansiedade materna e suas conseqüências fetais. O momento adequado da analgesia é aquele que a parturiente julga necessário e a técnica deve ser adaptada ao momento em que a analgesia foi solicitada.

A amniotomia deve ser tardia, ou seja, com dilatação cervical de mais de $8 \mathrm{~cm}$. Recomendase tal atitude pelo efeito protetor da bolsa das águas sobre a cabeça fetal. Deve ser lembrado que no TPP a progressão da dilatação pode ser muito rápida. O colo pode estar dilatado para 4-5 cm e atingir dilatação total em apenas uma hora. Além disso, no caso de prematuros o colo nem sempre necessita estar com $10 \mathrm{~cm}$ de dilatação para ocorrer o nascimento. O despreendimento do polo cefálico e do bisacromial deve ser lento para se evitarem os traumas sobre o sistema nervoso central e o plexo braquial, além de favorecer a expressão torácica durante a passagem pelo canal de parto, permitindo uma expansão pulmonar adequada. Daí, a importância da equipe, que deve estar atenta a estes cuidados.

A episiotomia deve ser ampla o suficiente para permitir o nascimento sem resistência perineal.

Apesar de o fórcipe baixo encurtar o período expulsivo e diminuir a incidência de hemorragia do sistema nervoso central, não o utilizamos quando o peso fetal estimado for inferior a $1.500 \mathrm{~g}$, situação em que seu emprego pode ser danoso ao feto.

Indicamos a via abdominal diante de intercorrências clínicas e obstétricas clássicas e nas apresentações cefálicas defletidas e pélvicas, situações em que os traumas fetais prejudicam o prognóstico neonatal. A operação cesariana nem sempre se traduz em extração fetal fácil. Na realização da histerotomia prefere-se a incisão transversa sempre que possivel mas, se o segmento estiver mal preparado, a extração fetal é mais difícil e, neste caso, deve-se realizar a incisão segmento-corporal longitudinal. 
A laqueadura do cordão umbilical deve ser realizada após 45 a 60 segundos, mantendo-se o recém-nascido em nivel inferior ao da placenta, sem praticar a ordenha. Este intervalo de tempo é necessário, pois sabe-se que $50 \%$ do sangue do prematuro encontra-se na placenta contra 30\% no recém-nascido de termo. Por outro lado, a passagem exagerada de sangue para o recém-nascido pode levar à hiperbilirrubinemia e hiperviscosidade sangüínea.

Em relação à anestesia para a cesárea prefere-se a raquianestesia com agulha fina em que se utiliza menor massa de concentração de anestésico, com menor comprometimento fetal. Devese tomar cuidados com as gestantes que foram submetidas à tocólise recente (com beta-adrenérgicos, sulfato de magnésio ou antagonistas do cálcio), pela possibilidade de interação medicamentosa com as drogas anestésicas, podendo levar a hipertensão arterial. Recomenda-se que após a suspensão da tocólise se dê um intervalo de pelo menos duas horas para se fazer a anestesia.

\section{Referências}

1. Cunningham FG, Leveno KJ, Bloom SL, Hauth JC, Gistrap III LC, Wenstrom KD. Williams obstetrics. 22nd ed. New York: McGraw-Hill; 2005. p. 855-80.

2. Iams JD, Creasy RK. Preterm labor and delivery. In: Creasy RK, Resnik R, Iams JD, editors. Maternal-fetal medicine: principles and practice. 5 th ed. Philadelphia: Saunders; 2004. p. 623-61.

3. Iams JD. Preterm birth. In: Gabbe SG, Niebyl JR, Simpson JL, editors. Obstetrics: normal and problem pregnancies. 4th ed. Philadelphia: Churchill Livingstone; 2002. p. 755-826.

4. American Academy of Pediatrics. American College of Obstetricians and Gynecologists. Guidelines for perinatal care. 4th ed. Elk Grove Village: AAP/ACOG; 1997.

5. Leitich H, Egarter C, Kaider A, Hohlagschwandtner M, Berghammer P, Husslein P. Cervicovaginal fetal fibronectin as a marker for preterm delivery: a metaanalysis. Am J Obstet Gynecol. 1999;180(5):1169-76.

6. Bittar RE, Yamasaki AA, Sasaki S, Zugaib M. Cervical fetal fibronectin in patients at increased risk for preterm delivery. Am J Obstet Gynecol. 1996;175(1):178-81.

7. Helfgott AW, Willis DC, Blanco JD. Is hydratation and sedation beneficial in the treatment of threatened preterm labor? A preliminary report. J Matern Fetal Med. 1994;3(1):37-42.

8. Moutquin JM, Sherman D, Cohen H, Mohide PT, Hochner-Celnikier D, Fejgin M, et al. Double-blind, randomized, controlled trial of atosiban and ritodrine in the treatment of preterm labor: a multicenter effectiveness and safety study. Am J Obstet Gynecol. 2000;182(5):1191-9.
9. European Atosiban Study Group. The oxytocin antagonist atosiban versus the â-agonist terbutaline in the treatment of preterm labor. A randomized, double-blind, controlled study. Acta Obstet Gynecol Scand. 2001;80(5):413-22.

10. Worldwide Atosiban versus Beta-agonists Study Group. Effectiveness and safety of the oxytocin antagonist atosiban versus beta-adrenergic agonists in the treatment of preterm labour. BJOG. 2001;108(2):133-42.

11.Papatsonis DN, Van Geijn HP, Ader HJ, Lange FM, Bleker OP, Dekker GA. Nifedipine and ritodrine in the management of preterm labor: a randomized multicenter trial. Obstet Gynecol. 1997;90(2):230-4.

12. Macones GA, Berlin M, Berlin JA. Efficacy of oral betaagonist maintenance therapy in preterm lator: a meta-analysis. Obstet Gynecol. 1995;85(2):313-7.

13. da Fonseca EB, Bittar RE, Carvalho MH, Zugaib M. Prophylactic administration of progesterone by vaginal suppository to reduce the incidence of spontaneous preterm birth in women at increased risk: a randomized placebo-controlled double-blind study. Am J Obstet Gynecol. 2003;188(2):419-24.

14. Sanchez-Ramos L, Kaunitz AM, Delke I. Progestational agents to prevent preterm birth: a meta-analysis of randomized controlled trials. Obstet Gynecol. 2005; 105(2):273-9.

15. Kenyon SL, Taylor DJ, Tarnow-Mordi W; ORACLE Collaborative Group. Broad-spectrum antibiotics for spontaneous preterm labor: the ORACLE II randomised trial. Lancet. 2001;357(9261):989-94.

16. Schrag S, Gorwitz R, Fultz-Butts K, Schuchat A. Prevention of perinatal group B streptococcal disease. Revised guidelines from the CDC. MMWR Recomm Rep. 2002;51(RR-11):1-22.

17. Liggins GC, Howie RN. A controlled trial of antepartum glucocorticoid treatment for prevention of the respiratory distress syndrome in premature infants. Pediatrics. 1972;50(4):515-25.

18. Effect of corticosteroids for fetal maturation on perinatal outcomes. NIH Consensus Development Panel on the Effect of Corticosteroids for Fetal Maturation on Perinatal Outcomes. JAMA. 1995;273(5):413-8.

19. National Institutes of Health. Antenatal corticosteroids revisited: repeat courses [conference on the Internet]. Consensus Development Conference Statement; 2000 Aug 17-18; Bethesda; 2000 [cited 2005 Jun 10]. Available from: http:// c o n s e n s u s. nih.gov/2000/ 2000AntenatalCorticosteroidsRevisted 1 $12 \mathrm{html} . \mathrm{htm}$

20.Jobe AH, Soll RF. Choice and dose of corticosteroid for antenatal treatments. Am J Obstet Gynecol. 2004;190(4):878-81.

21. Spinillo A, Viazzo F, Colleoni R, Chiara A, Maria Cerbo R, Fazzi E. Two-year infant neurodevelopmental outcome after single or multiple antenatal courses of corticosteroids to prevent complications of prematurity. Am J Obstet Gynecol. 2004;191(1):217-24. 